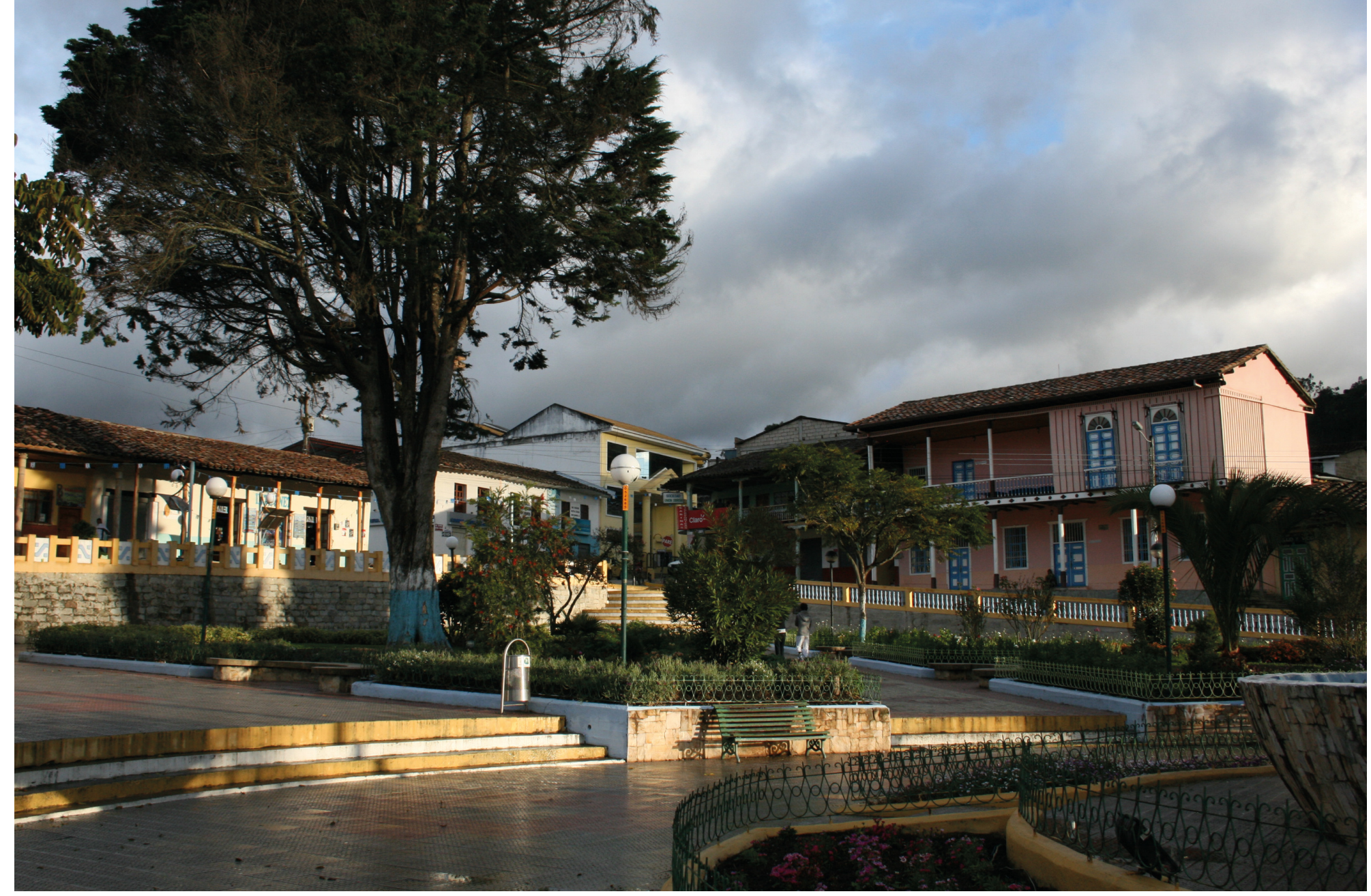

Figura 1. Plaza Central de Saraguro (Ecuador). Foto: Gonzalo Cerda Brintrup.

\title{
La integración de aprendizajes en la enseñanza de la planificación urbana articulada a contextos reales. Evaluación del proceso pedagógico en la formación del arquitecto a través de un caso de estudio
}

A integração da aprendizagem no ensino do planejamento urbano articulado aos contextos reais. Avaliação do processo pedagógico na formação do arquiteto através de um estudo de caso

The integration of learning in the teaching of urban planning aligned with real contexts: an evaluation of the pedagogical process in the training of architects through a case study

Fernando Pauta Calle

Docente de la Facultad de Arquitectura y Urbanismo, Universidad de Cuenca, Cuenca, Ecuador fernando.pautac@ucuenca.edu.ec | https://orcid.org/0000-0003-0826-6965

\section{Ximena Salazar}

Docente de la Facultad de Arquitectura y Urbanismo, Universidad de Cuenca, Cuenca, Ecuador | Candidata del Programa de Doctorado en Ordenamiento Territorial y Desarrollo Sostenible, Universidad Nacional de Cuyo, Mendoza, Argentina ximena.salazar@ucuenca.edu.ec | https://orcid.org/0000-0002-7486-8190

\section{Mónica González Llanos}

Docente de la Facultad de Arquitectura y Urbanismo, Universidad de Cuenca, Cuenca, Ecuador | Candidata del Programa de Doctorado en Ordenamiento Territorial y Desarrollo Sostenible, Universidad Nacional de Cuyo, Mendoza, Argentina monica.gonzalez@ucuenca.edu.ec | https://orcid.org/0000-0002-1031-2150 


\section{Resumen}

El artículo expone y evalúa el proceso pedagógico destinado a la formación específica en planificación urbana bajo la modalidad de itinerario académico, perteneciente a la Carrera de Arquitectura de la Universidad de Cuenca (Ecuador), en el que la vinculación con la sociedad o extensión universitaria representa una oportunidad integral para el aprendizaje en entornos reales. El plan de estudios pretende formar al estudiante en los ámbitos teóricos, metodológicos y técnicos necesarios para enfrentar la redacción o formulación de planes de ordenación urbanística. Para ello y respondiendo a la vez a una demanda concreta de los gobiernos locales, se trabaja en centros poblados menores a 3.000 habitantes, desde la premisa de "aprender planificación urbana, haciendo planificación urbana".

Como caso de estudio se expone el Plan de Ordenación de la Cabecera Parroquial de Urdaneta, Cantón Saraguro, formulado con un sólido soporte teórico conceptual y una rigurosidad técnica, que atendieron la necesidad presentada por la Municipalidad. La evaluación demuestra que alcanzar los resultados de aprendizaje relacionados a la formación en ordenación urbanística requiere una integración plena y consolidada de las metodologías de la formulación de un plan con aquellas referidas al proceso pedagógico, en términos de estrategias, medios para la articulación horizontal entre asignaturas, definición y distribución de actividades; y modalidades de trabajo y evaluación. Ha de tenerse.

\section{Palabras claves}

Aprendizaje activo, método de enseñanza, pedagógica, planificación urbana, planes urbanísticos

\section{Resumo}

0 artigo apresenta e avalia o processo pedagógico focado para a formação específica do planejamento urbano baixo a modalidade de itinerário acadêmica da faculdade de arquitetura da universidade de Cuenca- Equador, no qual a vinculação com a sociedade ou estágio universitário apresenta uma oportunidade integral para o aprendizado em situações reais. 0 plano de estudo pretende ensinar ao estudante nas áreas teórica, metodologia e técnica, quais são necessárias para enfrentar a redação ou formulação de planos de ordenamento urbano. Para esse objetivo e respondendo de forma paralela a necessidade de governos municipais, trabalha-se em cidades com população menor a 3000 habitantes, baixo a premissa de aprender o planejamento urbano, fazendo planejamento urbano.

Como exemplo apresentase 0 planejamento urbano do bairro Urdaneta, do municipio de Saraguro, desenvolvido com um forte suporte teórico, conceitual e com rigorosidade técnica, o mesmo que cumpriu a necessidade apresentada pelo município.

As avaliações demonstram que para alcançar os resultados do aprendizado relacionados com a formação no ordenamento urbano precisa de uma integração completa e definida das metodologias para a formulação de um plano com aquelas referidas ao processo pedagógico, em termos de estratégias, médios para articulação horizontal entre as diferentes matéria, definições e distribuição de atividades; e modalidades de trabalho e avaliação. Deve-se dar atenção que a está imbricação representa o aspecto central para conseguir o resultado de aprendizagem.

\section{Palavras-chaves}

Aprendizado ativo; método de ensino; prática pedagógica; planejamento urbano; planos urbanos

\section{Abstract}

This article presents and evaluates the pedagogical process used in the urban planning training in the architecture degree program's curriculum at the University of Cuenca, Ecuador, in which the partnerships with society or university outreach represent a comprehensive opportunity to learn in real environments. The curriculum seeks to train students in the theoretical, methodological and technical fields required in the writing or formulation of urban plans. To this end, while responding to the specific demands of local governments, the program works in population centers of less than 3,000 inhabitants, under the premise of "learning urban planning, doing urban planning." The Urdaneta Urban Plan, Saraguro District, is presented as a case study. It was formulated with strong theoretical-conceptual support and technical rigor, which met the Municipality's needs. The evaluation shows that achieving the learning outcomes related to urban planning requires the full consolidated integration of the methodologies to develop a plan related to the pedagogical process, in terms of strategies, means to horizontally coordinate between classes, and determine and distribute activities; and manners of working and evaluation. It must be kept in mind that this interconnection is the main factor in achieving the learning outcomes.

\section{Keywords}

Active learning, teaching methods, teaching practice, urban planning, urban plan 
* Artículo basado en la evaluación del programa académico implementado en la Opción de Planificación Urbana durante el periodo académico septiembre 2018 - julio 2019, Facultad de Arquitectura y Urbanismo de la Universidad de Cuenca.

\section{Introducción*}

La universidad se encuentra inmersa en una sociedad que experimenta cambios constantes, enfrenta grandes incertidumbres y procesos multidimensionales y multiescalares cada vez más complejos. Surge entonces la necesidad de superar la vigencia de modelos educativos mecanicistas y reduccionistas que bloquean la visión holistica de esta realidad, para avanzar a un aprendizaje autorreflexivo, en el que los estudiantes estén expuestos a diversos puntos de vista y alentados continuamente a desafiar sus propias suposiciones (Korobar y Siljanoska, 2016). El reto es consolidar la producción del conocimiento que emerja de realidades concretas, complejas y con problemáticas diversas, centrando el modelo educativo y sus respectivos planes de estudio en el aprendizaje.

Concretamente, respecto a la formación en planificación urbana, ésta se encuentra influenciada por diversos factores asociados a la cultura de planificación nacional, las tendencias de urbanización, aspectos socioeconómicos, entre otros, por lo que los programas y planes de estudio muestran diferencias considerables de contenido y formato (Frank y Silver, 2018). Sin embargo, las propuestas pedagógicas de las universidades tienen en común una creciente reorientación hacia la concreción de su responsabilidad social territorial transformadora (Molina, 2015), un acercamiento a problemáticas sociales (Sánchez de Matrama, 2004) y una ampliación de la cobertura de la educación respecto de sus beneficiarios (Henríquez, 2018), en aras de una mayor vinculación con problemáticas reales en ámbitos específicos. Es así como, en el contexto de un enfoque constructivista, se ha dado una permanente búsqueda de estrategias orientadas precisamente a un aprendizaje activo y comprometido en el que el alumno alcanza un conocimiento a través de experiencias directas en contextos auténticos (Baldwin y Rosier, 2017; Frank y Silver, 2018). En este sentido, diversas experiencias han demostrado que la participación de los estudiantes en proyectos de alcance comunitario resulta una herramienta de enseñanza efectiva (Teal, Huang y Rodiek, 1998). No obstante, el principal problema que se ha afrontado ha sido su permanencia y réplica en el tiempo (Menéndez y Tarabella, 2017).

Paralelamente, lo expuesto exige interrogarse en cuánto las propuestas pedagógicas concretan dichos modelos y permiten alcanzar un aprendizaje sustantivo, considerando que, aunque se trate de modelos y planes muy bien elaborados, entre ellos y los resultados de aprendizaje pueden mediar importantes diferencias.

En este marco y con el fin de aportar al debate, el artículo expone y evalúa el proceso pedagógico destinado a la formación específica en planificación urbana bajo la modalidad de itinerario académico de la Carrera de Arquitectura de la Universidad de Cuenca (Ecuador), en el que la vinculación con la sociedad o extensión universitaria representa una oportunidad única para el aprendizaje en entornos reales.

\section{Metodología}

En un primer momento se presenta una breve caracterización del itinerario académico, cómo está concebido en el marco del plan de estudios de la carrera y los resultados de aprendizaje que persigue. A continuación, se expone la propuesta pedagógica que muestra 
la articulación entre la metodología seguida para la elaboración de un plan urbanístico -que el estudiante aplicará en el ejercicio profesional- y las estrategias didácticas planteadas. La evaluación se realiza a través del análisis de un caso de estudio, cuya descripción se desarrolla en términos del proceso pedagógico y los productos que en cada etapa han generado los estudiantes.

El referente para reflexionar sobre el impacto que causa en los estudiantes las estrategias de la propuesta pedagógica lo constituyen los resultados de aprendizaje del Taller, espacio en el que se da la síntesis de los aprendizajes de las demás asignaturas. En términos cuantitativos, se considerará la evaluación realizada a los estudiantes a través de una rúbrica en concordancia con los mencionados resultados de aprendizaje.

\section{El itinerario académico de planificación urbana}

En el Ecuador, al igual que en casi toda Latinoamérica, el sistema territorial ha experimentado un modelo de desarrollo cuyas consecuencias han derivado en grandes desequilibrios e inequidades (Pauta, 2013). Al respecto, la cobertura de las reflexiones y trabajos que abordan la realidad de lo urbano con frecuencia han seguido esta tendencia, centrándose preferentemente en metrópolis y ciertos centros urbanos intermedios (Bermúdez et al., 2016; Kanai, Grant y Jianu, 2018). Más allá de esas grandes y medianas centralidades, existe una extensa red de pequeños centros poblados que en muchos casos constituyen, por sus funciones, eslabones de conexión entre las ciudades y el entorno rural (Capel, 2009) que están generalmente fuera del foco de atención de los diálogos de planificación y políticas, alejados de la toma de decisiones y que luchan por insertarse en las agendas de trabajo de los organismos decisorios (Rumbach, 2016). La universidad, en ese sentido, cumple un doble rol: da respuesta al requerimiento de estos planes en centros urbanos de tamaños bastante inferiores a lo calificado como ciudad, $y$, paralelamente, forma profesionales en el ámbito de la planificación urbana.

En este contexto, la Carrera de Arquitectura de la Universidad de Cuenca ha planteado, desde 1987, en las diferentes versiones de sus planes de estudio el fortalecimiento de la formación profesional en ámbitos específicos, creando tres opciones curriculares de carácter vocacional en el último año. El estudiante elige una de las áreas en función de sus propios intereses, decidiendo de qué manera desea completar su instrucción. En el actual Reglamento de Régimen Académico (2019), estas opciones constituyen itinerarios académicos configurados sobre la integración de conocimientos teórico disciplinares, metodológicos y tecnológicos.

En particular, el Itinerario u Opción de Planificación Urbana constituye una propuesta académica coherente que configura un ambiente de aprendizaje en el que sucede con creatividad y rigurosidad la integración de la teoría con la práctica. Con contenidos en constante actualización, este itinerario se ha retroalimentado con la incorporación de nuevos planteamientos y herramientas relativas a la planificación urbanística, convirtiendo cada ejercicio en una reflexión para los docentes que los lleva a sumirse en un "bucle reflexivo ilimitado" (Ruiz, 2002, p. 20).

La interacción universidad-comunidad-gobiernos locales ha permitido incrementar el conocimiento mutuo, adquirir un compromiso 


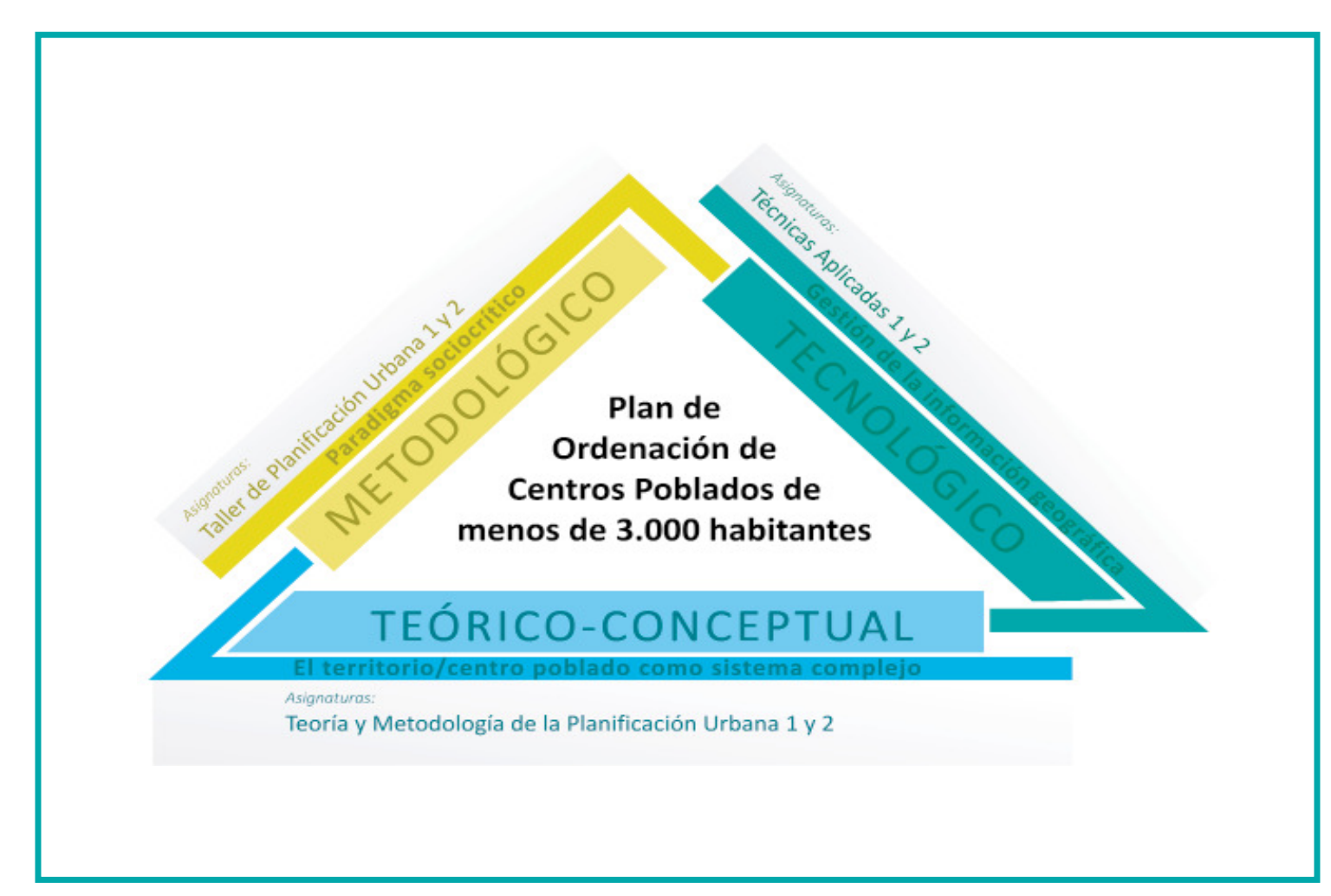

Figura 2. Modelo de integración curricular. Fuente: Elaboración de los autores.

con el desarrollo de estas localidades y generar sinergias. Situación que logra concretarse con la suscripción de convenios de cooperación interinstitucional con diferentes niveles de gobierno, que a la fecha y en total han viabilizado el trabajo en 47 cabeceras parroquiales en las provincias de Azuay, Cañar, Morona Santiago y Loja.

Específicamente, se pretende formar al estudiante en los ámbitos teóricos, metodológicos y tecnológicos necesarios para enfrentar la formulación de planes de ordenación (Jaramillo, 2014), alcanzando los siguientes resultados de aprendizaje:

1. Aplica la metodología y las técnicas pertinentes en la elaboración de diagnósticos de planes de ordenamiento urbano sostenible de asentamientos menores de 3.000 habitantes.

2. Aplica la metodología y las técnicas pertinentes en la elaboración de sintesis y prognosis de planes de ordenamiento de las características señaladas.

3. Aplica la metodología y las técnicas pertinentes en la elaboración de la imagen objetivo de planes de ordenamiento de las características señaladas.

4. Aplica la metodología y las técnicas pertinentes en la elaboración de propuestas de planes de ordenamiento urbano sostenible de asentamientos menores de 3.000 habitantes.

5. Desarrolla proyectos urbanos y arquitectónicos derivados del plan de ordenamiento urbano sostenible, que alcanzan los niveles de idea y pre-factibilidad. 
Aprender Planificación Urbana, haciendo Planificación Urbana Vincular la formación profesional a metas colectivas como la ordenación de pequeños centros poblados menos de 3.000 habitantes (Jaramillo, 2014), busca impactar positivamente en estos lugares y en el futuro del estudiante. Se plantea así una propuesta pedagógica que cumple un doble propósito. Por una parte, está la elaboración misma del plan con un sólido soporte técnico como respuesta a peticiones concretas de distintos gobiernos locales; $y$, por otra, combinar estrategias pedagógicas que permitan al estudiante aprender haciendo e integrar conocimientos de distintas asignaturas, de manera tal que pueda aplicarlos con fundamento, en un ejercicio de ordenación urbana.

Un postulado inicial que orienta la propuesta es superar la inevitable fragmentación del conocimiento en asignaturas en el currículo (Córdoba, 2014), las cuales, si bien se articulan con relativa fluidez de forma vertical, afrontan diversas dificultades al establecer una vinculación horizontal, frente a una realidad que no es compartimentada. Ya se trate de una "gran" ciudad o un pequeño centro poblado, se requiere la integración de saberes para afrontar un proceso de ordenación (Figura 2).

En la Opción de Planificación Urbana se ha generado un modelo en el que cada asignatura, cumpliendo una función especifica, aporta a un currículo con pertinencia. Bajo un proceso dialéctico y sin perder su individualidad y alcances, sus contenidos y actividades se organizan de manera tal que consiguen armonización, inclusión, coordinación temporal y compartición, aspectos que según Harden (2000) constituyen peldaños básicos para un tránsito hacia la integración.

Ámbito teórico-conceptual

"La construcción física del territorio es inseparable de las técnicas (lo factible) y las leyes (lo válido) que la regulan, orientan y determinan en mayor o menor medida" (Ruiz, 2002, p. 67). Para alcanzar una intervención rigurosa, se requiere de una base teórica que posibilite el entendimiento del objeto de estudio y la argumentación misma del proceso metodológico a seguir en un ejercicio de planificación. Asimismo, es evidente entonces que con este abordaje es imperativo el conocimiento y reflexión del marco legal en materia de ordenación urbanística de una forma contextualizada, superando el frío repertorio de disposiciones vigentes (ibidem).

El soporte teórico fundamental para la práctica en el Taller y en el ejercicio profesional, lo cubren las asignaturas de Teoría de la Planificación Urbana 1 y 2 , en las que se exponen los conceptos clave para aproximarse a la compleja realidad del territorio y el entendimiento del rol que va a desempeñar su planificación.

\section{Ámbito tecnológico}

Las herramientas tecnológicas facilitan el manejo y procesamiento de la información, en la medida que permiten a los alumnos explorar y desarrollar su capacidad para visualizar espacialmente los fenómenos urbanos. En esta dirección, la asignatura Técnicas Aplicadas no se limita al manejo de un software, sino a la gestión misma de la información territorial, articulándose al Taller a través de tareas integradas que apoyan el desarrollo del plan de ordenación. 


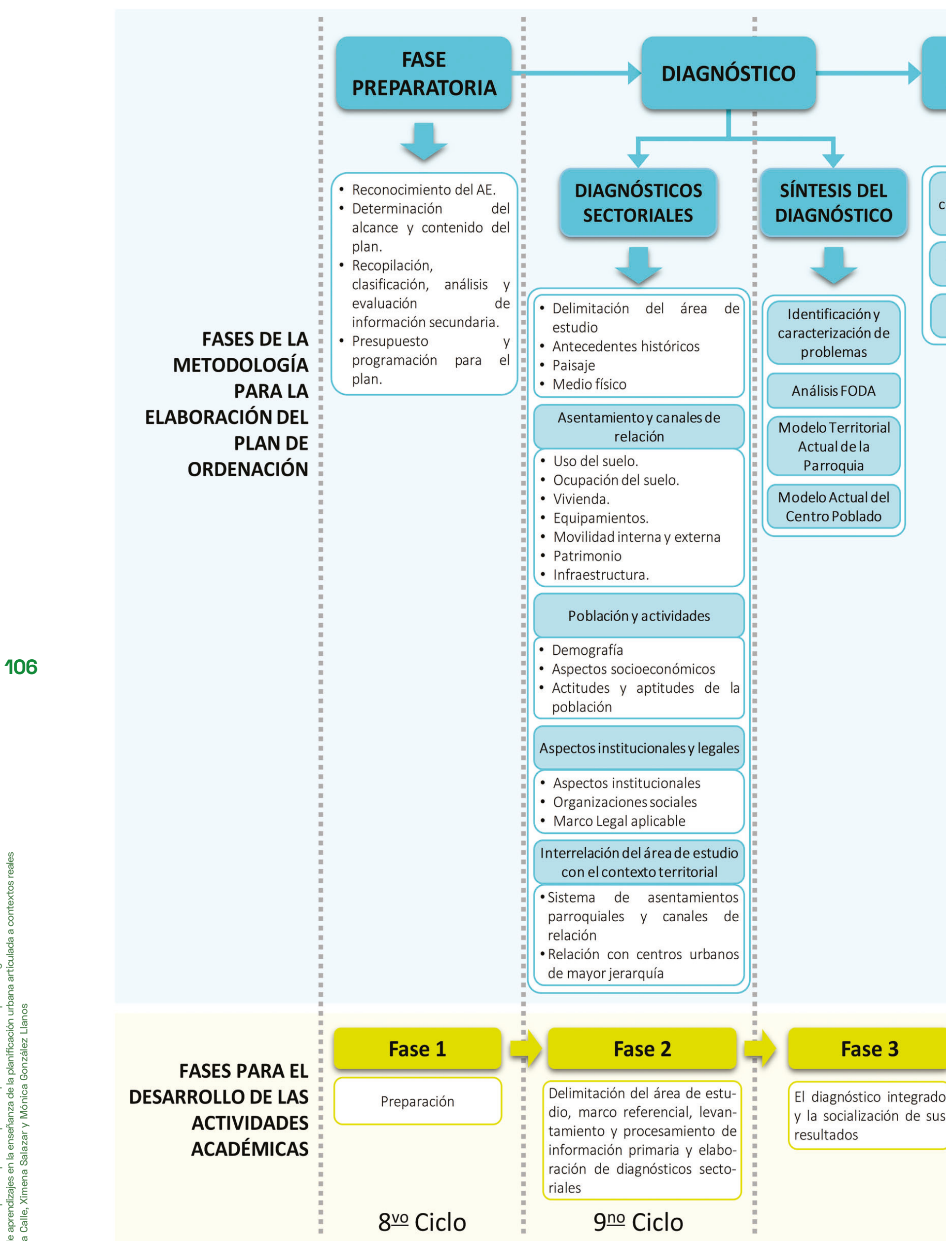

Figura 3. Proceso metodológico para la elaboración del Plan de Ordenación por periodo 


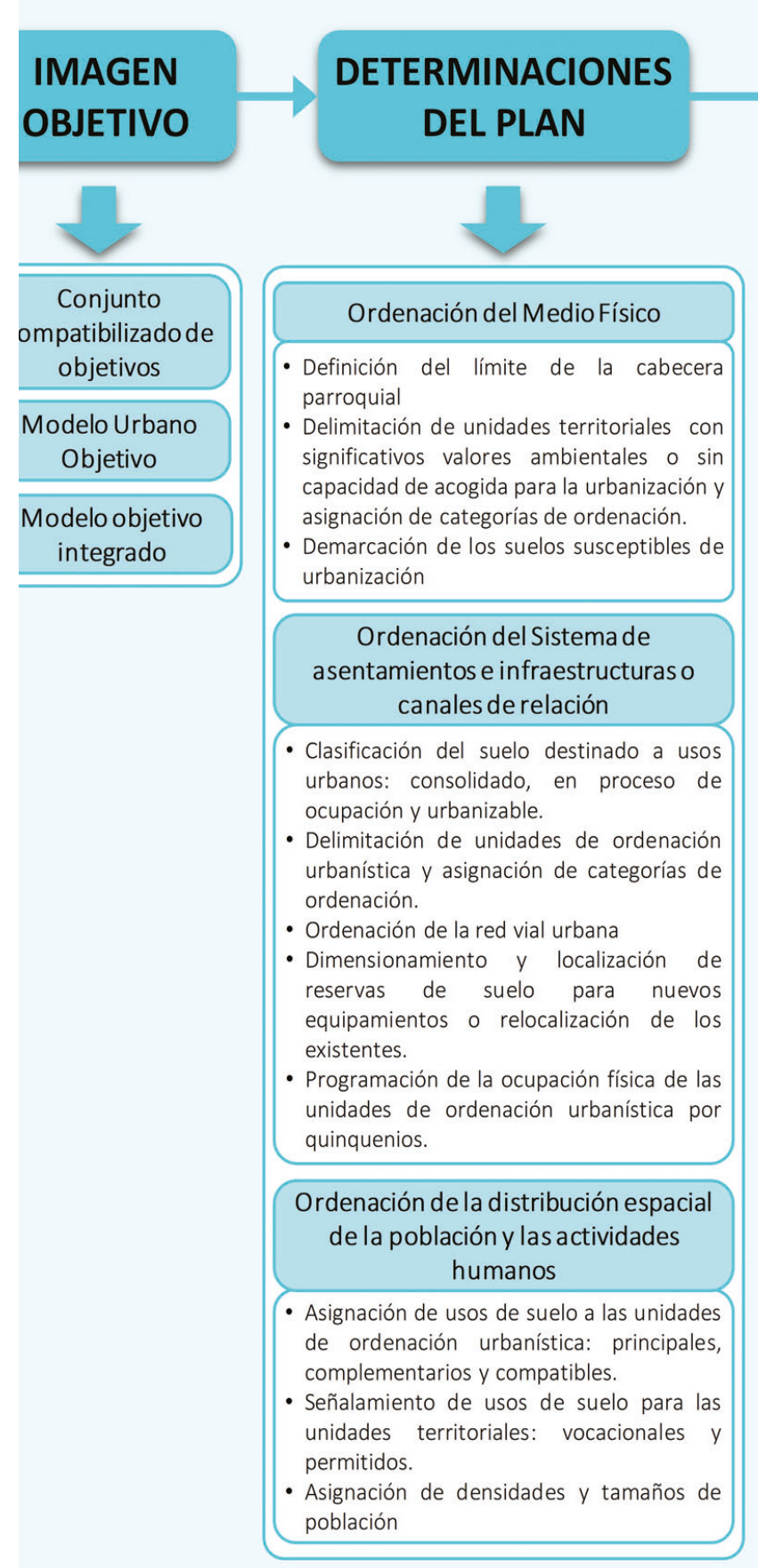

\section{NORMATIVA DE USO Y OCUPACIÓN DEL SUELO}

Fase 4

Construcción del modelo objetivo del centro poblado y formulación de las determinaciones del plan

\section{0ํㅡㄹ Ciclo}




\section{Ámbito metodológico}

En el Taller el profesor orienta y guía explícitamente el desarrollo de los contenidos del plan de ordenación, desde las fases iniciales hasta la formulación de propuestas de ordenación en términos de determinantes, normativas y proyectos. Se concreta un espacio en el que el ejercicio práctico permite una lectura de la realidad y el riesgo que supone el ejercicio de la decisión (Costa, Ochoa y Matos, 2015), desarrollando abstracciones que posibilitan la construcción de argumentos, y dando respuestas que, lejos de preconcepciones o asociación directa a situaciones conocidas, responden a la realidad de cada centro poblado.

De esta manera, a partir de una aproximación socio-crítica y etnográfica, prima un fuerte involucramiento de los estudiantes en la formulación del plan que será expuesto y entregado a la comunidad, como una contribución real a la sociedad, mediante lo cual se promueve además las mejores condiciones para el aprendizaje, en la medida en que los alumnos crean algo que va a ser compartido (Olmedo y Farrerons, 2017).

El aprendizaje fuera del aula, aspecto clave de este proceso, proporciona experiencia, a docentes y estudiantes, en la vinculación de los contenidos teóricos y metodológicos a diferentes regiones y entornos culturales, sensibilizándolos sobre la variedad de posibles enfoques y soluciones. El aprendizaje experiencial descansa principalmente en el estudiante -quien interrelaciona acción, reflexión, abstracción y aplicación- y se traduce en un cambio de actitud y conocimiento, consecuencia de la exposición a condiciones de la vida real (Korobar y Siljanoska, 2016), convirtiendo la realidad del territorio en un medio de enseñanza (García, Villar, Fraile, Sánchez y Márquez, 2018).

El trabajo docente se centra en llevar al estudiante a un manejo adecuado de la terminología urbanística, evitando incluso la asociación errónea de ciertos términos a aspectos y componentes urbanos concretos. Adicionalmente, en el Taller se enfatiza la enseñanza de estrategias de análisis para la lectura e interpretación de la realidad, en un escenario en el que el alumno comprenda la realidad integrando las diversas dimensiones que la estructuran y plantee soluciones con rigurosidad técnica. Por su parte, la participación de la población se promueve a través de la elaboración de cartografía social, de talleres, conversatorios, visitas de campo, entre otras actividades, que permiten disponer de la imagen colectiva que tienen los habitantes de sus territorios.

En términos operativos concretos, el proceso metodológico seguido para la formulación del plan de ordenación se articula a los periodos académicos (Figura 3) y, de forma horizontal, con las otras dos asignaturas del itinerario.

\section{Caso de estudio}

El Plan de Ordenación Urbanística de la Cabecera Parroquial de Urdaneta Urdaneta es una de las siete parroquias rurales del cantón Saraguro de la provincia de Loja en el sur del Ecuador. Según el VII Censo de Población, efectuado en 2010, contaba con 3.142 habitantes y su cabecera parroquial o centro poblado, con 546 . Su tasa de crecimiento en el último período inter-censal: 2001-2010, fue del 2,01\%. 
La economía parroquial se sustenta en la agricultura y ganadería tradicionales, con predominio del minifundio de modestos niveles de producción y productividad, destinándose sus excedentes al mercado cantonal. La cabecera parroquial constituye un centro de gestión y administración parroquial rural, de intercambio a nivel básico y de servicios públicos en ámbitos como la salud, educación y asistencia social.

Pese a sus más de sus 150 años de existencia, este centro poblado no había sido motivo de ningún plan de ordenación, de ahí la importancia de la presencia de la Opción de Planificación Urbana de la Carrera de Arquitectura, a fin de coadyuvar a que los gobiernos parroquial y cantonal cuenten con un instrumento que les permita cumplir sus competencias constitucionales vinculadas con la elaboración y gestión de dicho plan.

En este contexto y de conformidad con el diseño curricular de la carrera, la elaboración del Plan de Ordenamiento siguió las fases sustentadas a continuación y con un equipo de trece estudiantes:

\section{Fase 1: Preparación}

A las actividades de preparación descritas en la Figura 2 se suma una introducción preliminar a aspectos conceptuales básicos de la planificación urbana.

Fase 2: Delimitación del área de estudio, marco referencial, levantamiento y procesamiento de información primaria y elaboración de diagnósticos sectoriales

Esta etapa requirió el cumplimiento de las siguientes macroactividades:

1. Organización y programación del período académico y el proceso mismo de enseñanza aprendizaje.

2. Delimitación del Área de Estudio, integrada por el Área Específica de Planificación, esto es, la cabecera parroquial, y por el Área de Influencia, la que abarcó los límites políticoadministrativos de la parroquia Urdaneta, cuya superficie es de $118,3 \mathrm{~km}^{2}$.

La delimitación del Área Especifica de Planificación fue producto de una propuesta preliminar formulada en el aula con sustento en los elementos conceptuales impartidos por los profesores, y de un reconocimiento de campo que puso énfasis en las condiciones de uso y ocupación del suelo actuales, en las tendencias de crecimiento y en la exclusión del suelo propiamente rural, según fuera posible.

El área delimitada abarcó una superficie de 63,20 ha, estimándose una densidad de 11 hab./ha, la cual demostró que, con las expectativas de crecimiento poblacional a veinte años, podría acoger las demandas de suelo que pudiesen generar la vivienda y otras actividades socioeconómicas. Esta realidad demostraba, que la ordenación del centro poblado dominantemente debía centrarse en su consolidación más que en su expansión.

De forma complementaria, se determinaron unidades espaciales relativamente homogéneas en cuanto a su grado de consolidación urbana y al uso de suelo, para lograr que los diagnósticos alcanzaran mayor profundidad en relación 

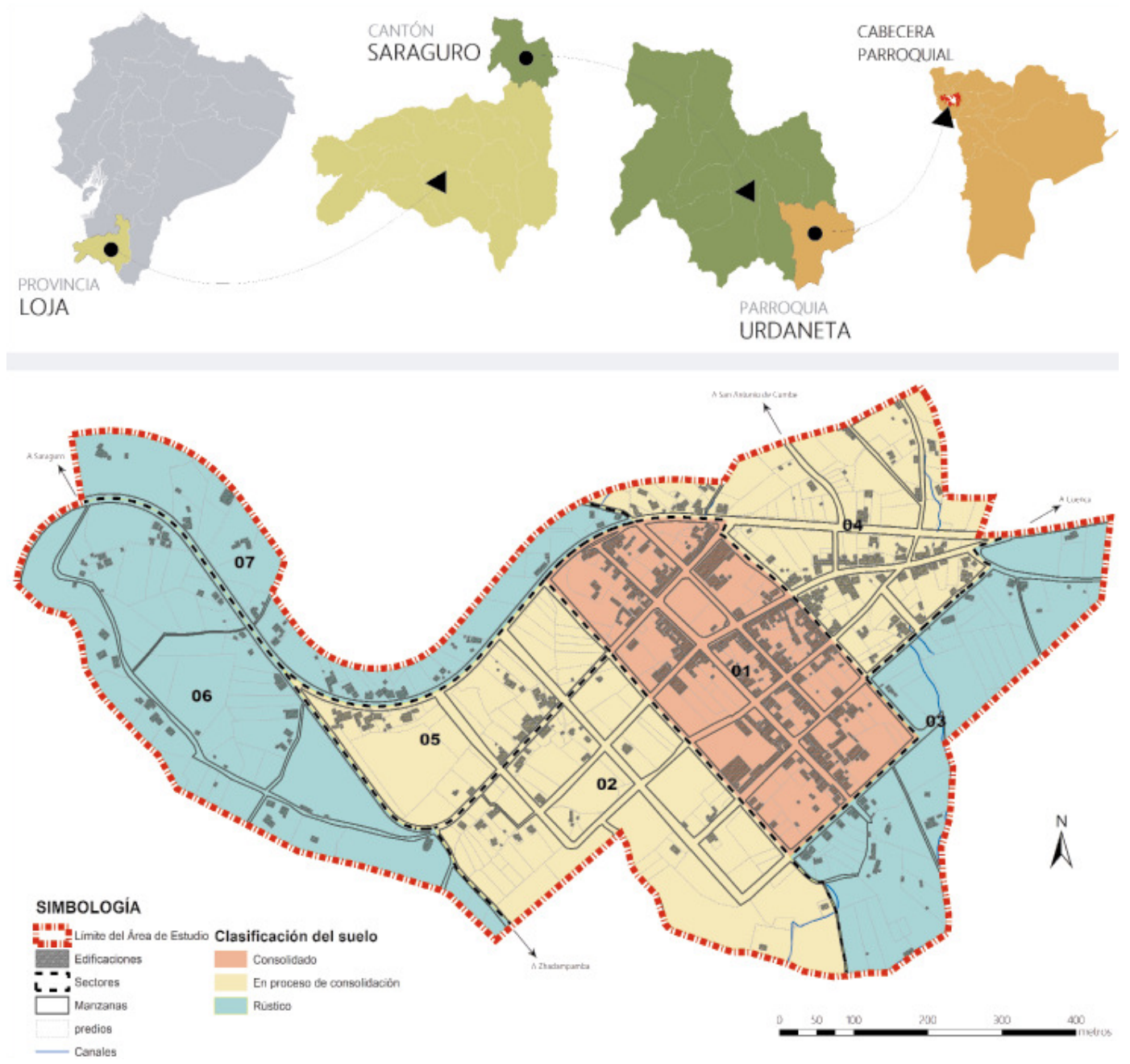

Figura 4. Ubicación, delimitación y sectorización del Área Especifica de Planificación. Fuente: Elaboración de los autores a partir de los datos del curso de la Opción de Planificación Urbana septiembre 2018 - julio 2019.

con la dimensión espacial de los temas abordados. Se definió entonces un sector consolidado -la centralidad-, cuatro sectores en proceso de ocupación y tres con suelo que muestra evidencias de ocupación rural (Figura 4). Al interior de los sectores se reconocieron las manzanas y sus predios como unidades de análisis especificas.

3. Establecimiento de marco referencial.

Esta actividad proveyó a los estudiantes la base conceptual, metodológica y operativa para la realización de cada uno de los diecinueve diagnósticos sectoriales, estableciendo: objetivos, definiciones operacionales, contenidos preliminares, metodología a seguir y determinación de la información primaria y secundaria a emplear.

4. Levantamiento de información.

En primera instancia, se diseñó el conjunto de instrumentos destinados al levantamiento de la información primaria requerida por los diagnósticos sectoriales (Tabla 1), elaborando en cada caso cuestionarios e instructivos de aplicación con las respectivas normas de aseguramiento de la calidad de la información. En un segundo momento, se difundió la elaboración del plan a la población para motivar su participación en el proceso.

Cumplidos estos pasos, vino la tarea de levantamiento de información. Para ello, los estudiantes debieron residir por el lapso de tres semanas en el centro poblado, a fin de cumplir la programación prevista y experimentando un fuerte vínculo 


\begin{tabular}{|l|l|c|}
\hline \multicolumn{1}{|c|}{ Instrumento } & \multicolumn{1}{|c|}{ Unidad } & $\mathbf{N}^{\circ}$ \\
\hline Censo predial & predio & 562 \\
\hline Censo de viviendas y hogares & viviendas & 190 \\
\hline Inventario vial & tramos viales & 119 \\
\hline Inventario de equipamientos comunitarios & equipamientos & 13 \\
\hline Censo de organizaciones sociales & $\begin{array}{l}\text { organizaciones } \\
\text { sociales }\end{array}$ & 5 \\
\hline $\begin{array}{l}\text { Diario de campo para el inventario y } \\
\text { valoración de unidades de paisaje }\end{array}$ & $\begin{array}{l}\text { unidades de } \\
\text { paisaje }\end{array}$ & 5 \\
\hline $\begin{array}{l}\text { Registro de bienes inmuebles de interés } \\
\text { cultural }\end{array}$ & edificaciones & 46 \\
\hline $\begin{array}{l}\text { Conteo vehicular en estaciones de ingreso- } \\
\text { salida del centro poblado }\end{array}$ & estaciones & 2 \\
\hline $\begin{array}{l}\text { Encuesta de aptitudes y actitudes de la } \\
\text { población }\end{array}$ & comercios & 55 \\
\hline $\begin{array}{l}\text { Inventario de anejos pertenecientes a la } \\
\text { parroquia }\end{array}$ & anejos & 11 \\
\hline
\end{tabular}

Tabla 1. Número de Instrumentos aplicados por tipo. Fuente: Elaboración de los autores a partir de los datos del curso de la Opción de Planificación Urbana septiembre 2018 - julio 2019.

con la realidad. Los profesores efectuaron visitas para cumplir labores de control, verificación y atención de inquietudes.

5. Procesamiento de información.

Una vez validada la información, se la procesó mediante un sistema de gestión de bases de datos, para la obtención de cuadros estadísticos e insumos para el análisis espacial. Precisamente, la expresión espacial de los fenómenos abordados en los diagnósticos sectoriales, requirió la producción mapas y gráficos como instrumentos esenciales para el análisis, conformando un sistema de información geográfica.

6. Elaboración de diagnósticos sectoriales.

Con sustento en la formación recibida, el conocimiento sensorial adquirido, la interpretación de la información disponible y el acompañamiento de los profesores, la siguiente etapa que desarrollaron los estudiantes fue examinar la realidad objetiva e inmanente ligada a cada una de las temáticas de los diagnósticos, conceptualizando y captando sus características esenciales, manteniendo una autorreflexión permanente acerca de lo que iban logrando aprehender de ella, y poniendo en juego su capacidad de problematización y abstracción. Se trató entonces, desde la perspectiva del estudiante, de un proceso intelectual en permanente evolución, reflejado en la producción del informe. 
Fase 3: El diagnóstico integrado y la socialización de sus resultados El propósito central de esta etapa fue pasar del análisis a la síntesis, buscando la explicación sistemática, global y detallada de las relaciones que articulan el fenómeno estudiado, esto es, el centro poblado como hecho real y concreto.

Para tal efecto, se aplicaron las herramientas impartidas en la formación teórica. Por una parte, la identificación y descripción de problemas fuertemente multidimensionales, para lo cual se usó formatos que describen sus atributos; seguido de la construcción de los respectivos árboles de problemas que explican sus causas y efectos. Metodológicamente y a fin de propiciar una visión integradora, esta sub-fase levantó los problemas en relación con los grandes elementos resultantes del entendimiento del centro poblado como sistema: medio físico, población y actividades, asentamientos e infraestructuras o canales de relación; y, marco institucional y normativo (Figura 2).

La problematización culminó con la formulación colectiva del "problema general", la sintesis más integradora, resultante de la vivencia de los estudiantes con la realidad y de las conclusiones derivadas de la interpretación crítica de la información. A ello se suma la aplicación del análisis FODA a los elementos antes identificados, para permitir que los alumnos visualicen las fortalezas y debilidades de la cabecera parroquial, las amenazas y oportunidades del entorno, y las líneas de acción válidas en la formulación del modelo objetivo y en las determinaciones del plan.

Productos esenciales del diagnóstico integrado constituyeron el Modelo Territorial Actual de la Parroquia, que muestra las tendencias actuales de uso y ocupación del suelo rural propiamente dicho, y el Modelo Actual del Centro Poblado, a partir de los submodelos referidos a cada uno de los grandes elementos del sistema. En una apretada sustentación de este modelo, se puede señalar que el centro poblado se emplaza en un suelo apto para la urbanización; no obstante, cerca del $60 \%$ de su superficie es susceptible a inundaciones por escorrentías superficiales provenientes de las formaciones naturales del costado noreste, y en la periferia se encuentran pequeñas zonas con susceptibilidad a movimientos en masa, inestables y de fuertes pendientes.

Desde varios puntos del perimetro se configuran cuencas visuales con significativos valores paisajisticos propios del medio rural andino. Se distingue en el asentamiento un núcleo central estructurado con una trama reticular rectangular que se enlaza a la vía interurbana que bordea la cabecera parroquial, y que se muestra significativamente consolidado; constituyendo una centralidad en el orden funcional, a la que le otorgan jerarquía los usos vinculados a la gestión y administración parroquial rural. Contiguos se hallan varios sectores en proceso de consolidación a los cuales se extiende la trama reticular, con diversidad de usos hacia el costado noreste; en tanto que hacia el noroeste y suroeste la función residencial pierde importancia, destacándose aquí la producción artesanal de bienes y, sobre todo, los usos rústicos y los lotes vacantes (Figura 4).

El tamaño de la población residente y las caracteristicas de la base económica parroquial no generan las condiciones mínimas de mercado para el emplazamiento de usos de mayor alcance y oferta, incluso de establecimientos vinculados a la prestación de servicios 
personales y afines a la vivienda, provocando fuertes dependencias de los centros urbanos más cercanos.

En algunas manzanas el suelo ha sido motivo de abuso por su intenso fraccionamiento y por la generación de lotes de formas irregulares. El parque edificado es predominantemente de dos pisos y su paisaje tradicional se ve alterado por actuaciones recientes que responden a la "arquitectura moderna". Varios bienes patrimoniales muestran abandono, mal estado de conservación e intervenciones inadecuadas. Asimismo, la mayoría de las viviendas evidencian regulares condiciones de habitabilidad y proporciones importantes de ellas son deficitarias por el número de dormitorios, cuartos y equipo sanitario. Varios equipamientos no cuentan con la infraestructura requerida, funcionan en locales arrendados y se carece de centros socioculturales y áreas verdes.

La red vial, en un importante número de tramos, no dispone de capa de rodadura y, en otros, el estado de esta infraestructura es apenas regular. La calidad del agua potable no cumple las normas recomendadas y tanto la cobertura de la red de alcantarillado como el servicio de recolección de basuras son parciales, incluso en el núcleo central. Pese a las ya mencionadas relaciones de dependencia de los centros mayores, se carece de transporte público.

Culminó esta fase con el desarrollo de un taller de socialización de los resultados de diagnóstico al que asistieron moradores del centro poblado y autoridades locales y cantonales. Los estudiantes sustentaron las conclusiones usando estrategias comunicativas apropiadas, con énfasis en sus implicaciones futuras bajo la hipótesis de la ausencia de acciones para alterar especialmente el curso de las tendencias negativas. Se dio paso luego a un proceso interactivo de preguntas y respuestas, que, además de atender las inquietudes ciudadanas, permitió validar el trabajo y recibir valiosas contribuciones para ajustar los informes.

Este evento comprendió también el pronunciamiento libre de los participantes de cara a un futuro más positivo para la cabecera parroquial como también más incluyente respecto a sus deseos y aspiraciones de desarrollo, a partir de lo cual se consiguió un insumo de gran importancia dado por el sujeto de la planificación, para la siguiente fase.

Fase 4: Construcción del modelo objetivo del centro poblado y formulación del plan de ordenación urbanística

Esta constituyó la fase de planificación misma y sus componentes son los siguientes:

1. Construcción del modelo objetivo

Con base en los resultados del diagnóstico, en la visión del proceso de evolución del centro poblado y en las determinaciones y directrices del Plan de Desarrollo y Ordenamiento Parroquial Rural de Urdaneta, se diseñó y expresó gráficamente la imagen del centro poblado a alcanzarse al año horizonte: 2039.

Formulación de un conjunto compatibilizado de objetivos.

Concebido como un cúmulo de pretensiones destinadas al mejoramiento de la calidad de vida de la población, los objetivos se elaboraron buscando expresar la voluntad de resolver los problemas actuales, prevenir los futuros, 


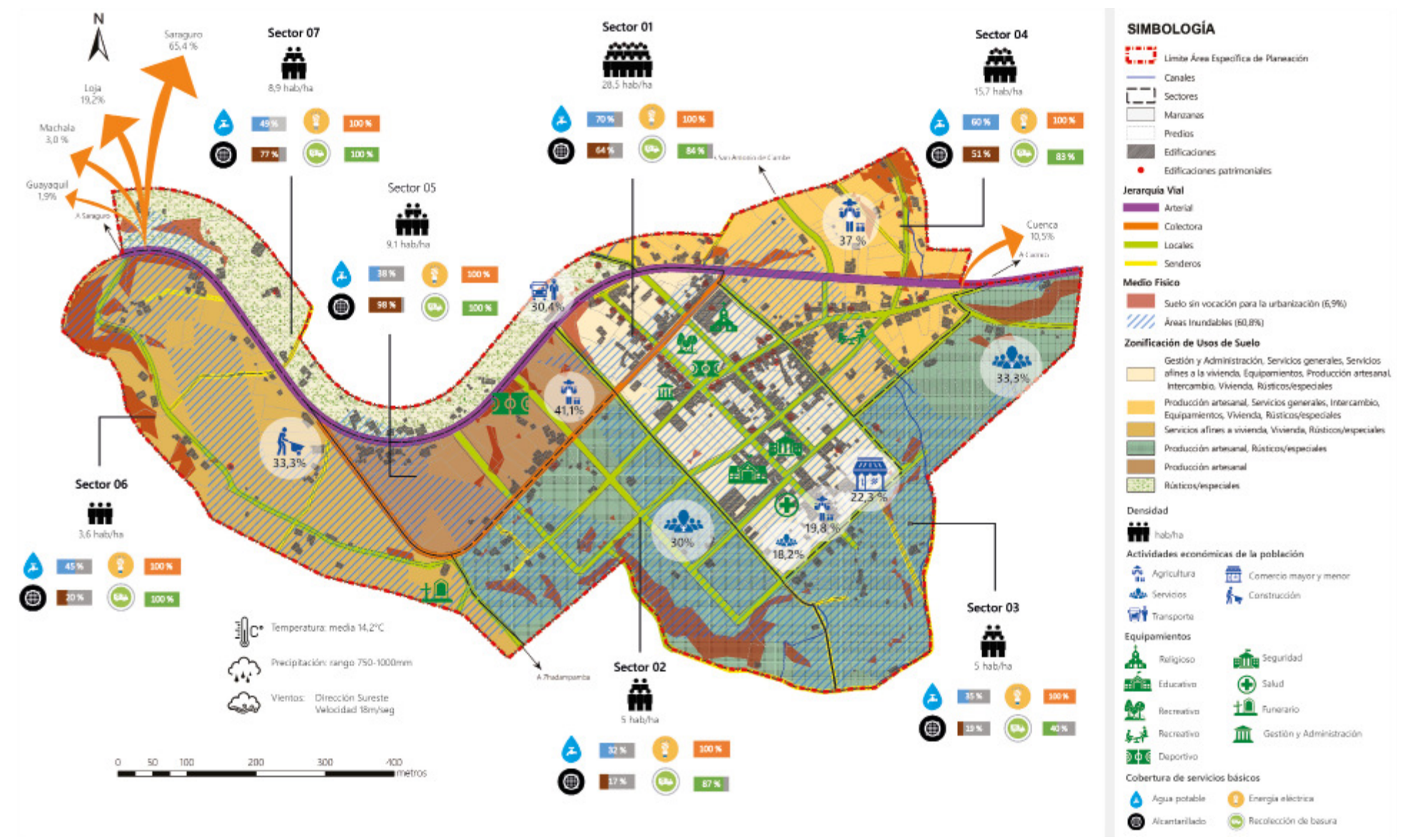

Figura 5. Modelo urbano actual de la Cabecera Parroquial de Urdaneta. Fuente: Elaboración de los autores a partir de los datos del curso de la Opción de Planificación Urbana septiembre 2018 - julio 2019.

aprovechar las tendencias positivas y las oportunidades del entorno, satisfacer las demandas, aspiraciones y deseos de desarrollo de la población; y cumplir las determinaciones de rango superior.

Elaboración del modelo objetivo por grandes elementos del centro poblado.

De modo análogo a la construcción del modelo actual y con fines de aprendizaje de los estudiantes, se construyeron en forma previa los sub-modelos de ordenación del medio físico, asentamientos e infraestructuras o canales de relación y distribución espacial de la población y actividades.

Formulación del modelo objetivo integrado.

Producto de los modelos precedentes se obtiene la expresión espacial integrada de los objetivos. En una ajustada síntesis, se puede manifestar que en general el centro poblado, en los próximos veinte años, deberá consolidar los asentamientos que lo conforman, más si su capacidad de recepción de población supera en mucho a la esperada en dicho horizonte. Se exceptúan de esta condición los suelos sin aptitud para la urbanización, en los cuales es necesario detener las tendencias de ocupación actuales. Las inundaciones por escorrentías superficiales se evitarán mediante la construcción de un canal de coronación que las conduzcan a los cuerpos de agua cercanos. A su vez, los puntos generadores de cuencas visuales desde y hacia el centro poblado, con sus espacios circundantes, serán motivo de control 


\begin{tabular}{|c|c|c|c|c|c|c|c|}
\hline \multirow{3}{*}{ Resultado de aprendizaje } & \multicolumn{7}{|c|}{ Calificaciones [\%] } \\
\hline & \multicolumn{3}{|c|}{ Tareas Individuales } & \multicolumn{3}{|c|}{ Tareas Grupales } & \multirow{2}{*}{$\begin{array}{c}\text { Tareas } \\
\text { Colectivas }\end{array}$} \\
\hline & minima & media & máxima & minima & media & máxima & \\
\hline $\begin{array}{l}\text { Aplica la metodologia y las técnicas pertinentes en } \\
\text { la elaboración de diagnósticos de planes de } \\
\text { ordenamiento urbano sostenible de asentamientos } \\
\text { menores de } 3.000 \text { habitantes. }\end{array}$ & 66 & 85 & 98 & 100 & 100 & 100 & 100 \\
\hline $\begin{array}{l}\text { Aplica la metodologia y las técnicas pertinentes en } \\
\text { la elaboración de sintesis y prognosis de planes de } \\
\text { ordenamiento de las caracteristicas señaladas. }\end{array}$ & 72 & 85 & 100 & 80 & 89 & 100 & 100 \\
\hline $\begin{array}{l}\text { Aplica la metodologia y las técnicas pertinentes en } \\
\text { la elaboración de la imagen objetivo de planes de } \\
\text { ordenamiento de las características señaladas. }\end{array}$ & 71 & 80 & 89 & 80 & 80 & 80 & 90 \\
\hline $\begin{array}{l}\text { Aplica la metodologia y las técnicas pertinentes en } \\
\text { la elaboración de propuestas de planes de } \\
\text { ordenamiento urbano sostenible de asentamientos } \\
\text { menores de } 3.000 \text { habitantes. }\end{array}$ & 87 & 95 & 100 & - & - & - & 100 \\
\hline $\begin{array}{l}\text { Desarrolla proyectos urbanos y arquitectónicos } \\
\text { derivados del plan de ordenamiento urbano } \\
\text { sostenible, que alcanzan los niveles de idea y pre- } \\
\text { factibilidad. }\end{array}$ & 83 & 92 & 98 & - & - & - & - \\
\hline
\end{tabular}

Tabla 2. Calificaciones promedio obtenida por los estudiantes por modalidad de trabajo y según resultados de aprendizaje. Fuente: Fuente: Elaboración de los autores a partir de los registros de calificaciones del curso de la Opción de Planificación Urbana septiembre 2018 - julio 2019.

a fin de evitar actuaciones que lleven a destruir su potencial.

El núcleo de la cabecera parroquial de Urdaneta deberá alcanzar la consolidación total y robustecer su condición de centralidad de gestión y administración, de intercambio, servicios, de emplazamiento de los equipamientos y de vivienda. Por su parte, la consolidación de los actuales sectores en proceso de ocupación como asentamientos de vivienda, equipamientos y servicios, alcanzará la mayor prioridad, a fin de superar la concentración-dispersión de los usos de suelo que muestran al momento. Se plantea un crecimiento compacto del centro poblado que optimice el aprovechamiento de las infraestructuras, los servicios básicos y los equipamientos disponibles, como asimismo los que se doten a futuro.

En el plano económico, se espera que las estrategias del plan de desarrollo parroquial generan las condiciones mínimas de mercado para el emplazamiento de usos de mayor alcance y oferta, que atenúen la dependencia de los centros urbanos más cercanos.

A través dela normativa reguladora, se precautela la compatibilidad y comportamiento que mantienen los usos de suelo, deteniendo las tendencias negativas de fraccionamiento de este recurso, así como su ocupación con edificaciones al margen de condiciones urbanisticas mínimas y sin integración al paisaje y al parque edificado tradicional. 
El patrimonio cultural edificado, será recuperado y conservado mediante medidas normativas y de inversión, acogiendo usos y actividades compatibles con sus caracteristicas.

Serán tareas prioritarias: la ampliación de la red de alcantarillado y del servicio de recolección de desechos sólidos, el mejoramiento de la calidad del agua potable y la dotación del servicio de transporte público a otros centros urbanos.

La vivienda deberá garantizar adecuadas condiciones de habitabilidad a la población y la dotación de cuartos, dormitorios y equipo sanitario. Se proveerá de los siguientes equipamientos: coliseo, mercado, camal, biblioteca y centro de bienestar social, y, con prioridad, áreas verdes y espacios de recreación activa. La plaza central, el subcentro de salud y el cementerio serán mejorados y la unidad de policía comunitaria se relocalizará.

Finalmente, se optimizarán las características geométricas de las vias, priorizándose la circulación peatonal, en el marco de una adecuada jerarquización funcional. Su ampliación, desde este mismo enfoque, será la indispensable, sobre todo en los sectores que mantendrán formas de ocupación más rurales.

2. Determinaciones del plan

El propósito central del plan elaborado fue concretar el modelo objetivo propuesto para el largo plazo, definiendo los elementos fundamentales de la estructura general adoptada para la ordenación del centro poblado y las condiciones específicas de uso y ocupación del suelo de las unidades espaciales establecidas para el efecto. Se formularon las determinaciones para la ordenación del medio físico, los asentamientos e infraestructuras o canales de relación y la distribución espacial de la población y las actividades humanas, de conformidad con los contenidos expuestos en la Figura 2.

3. Normativa de uso y ocupación del suelo

Para transitar del modelo actual al modelo objetivo es necesario regular las actuaciones previstas por el plan en cuanto a los usos de suelo, su ocupación o aprovechamientos y a los comportamientos de las actividades humanas permitidas. Desde una perspectiva muy ilustrativa, se pretende que el estudiante aprenda que, en materia de ordenación de la ciudad, tan importante es lo que se permite, como lo que no se permite. En ese marco, se formularon las normas aludidas en la Figura 2.

4. Programas, subprogramas y proyectos

La consecución del modelo objetivo previsto demanda, además, un conjunto de inversiones públicas, privadas y comunitarias (Figura 6), que debe ser ejecutado considerando la prioridad, la disponibilidad de recursos financieros y los beneficios sociales o económicos que generen. En su formulación, los estudiantes ponen en práctica su formación en diseño arquitectónico, construcciones, diseño urbano, presupuestos y programación de proyectos. 


\section{Resultados y discusión}

Centrando el interés de este acápite en la evaluación de los resultados de aprendizaje, en función de las rúbricas diseñadas para el efecto, y en sus expresiones cuantitativas, debe señalarse:

- Las mayores calificaciones que obtienen los alumnos se dan en las tareas grupales y colectivas, variando entre 80 y 100 , lo cual evidencia la importancia del trabajo en equipo que permite superar debilidades a nivel individual. Las menores calificaciones en estas tareas estarían asociadas a la complejidad que entrañan los resultados de aprendizaje vinculados con la elaboración de la síntesis del diagnóstico y prognosis, así como a la construcción de la imagen objetivo, por la abstracción con la que deben ser tratados.

- Las menores notas en las tareas individuales se explicarian en las dificultades que enfrentan los alumnos tanto por iniciarse en la formación de una disciplina que tiene por objeto de estudio fenómenos espaciales a mayor escala, cuanto por la adopción de nuevas formas de comunicación, particularmente la elaboración de informes técnicos. No obstante, estas dificultades al parecer son superadas conforme avanza la formación, pues los resultados de aprendizaje posteriores a la fase de diagnóstico muestran mejores calificaciones individuales.

- Las calificaciones individuales también revelan la necesidad de un acompañamiento más intenso del equipo de profesores a los estudiantes, asi como la creación de espacios para un debate más formativo, cuestiones que entrañan incrementos de horas de clase que no siempre podrán ser atendidos. De hecho, no debe descartarse el empleo de nuevos recursos educativos.

- Pese a lo expuesto, el análisis de los resultados de aprendizaje evidencia que el proceso pedagógico establecido para la Opción de Planificación Urbana, sobre la base de las estrategias adoptadas y su plena consolidación académica, logra formar a los estudiantes en la ordenación de centros poblados pequeños, en el marco de la teoría y práctica aplicable a la ciudad en general.

- La experiencia vivida en el transcurso de apenas 32 semanas lectivas, de conformidad con el Plan de Estudios, demuestra a los alumnos que el trabajo en equipo debidamente coordinado y ejecutado, es una necesidad en la planificación de la ciudad, pero a la vez una potencialidad.

- La actividad sustantiva de la vinculación con la sociedad y gobiernos locales representa una oportunidad colectiva para el aprendizaje, una posibilidad sociológica de interacción, derivada del logro del entendimiento cultural de los centros poblados intervenidos. Los alumnos logran vivir una práctica preprofesional con un fuerte compromiso social e institucional, que sin duda orientará su futuro ejercicio profesional.

- Estas actividades son de suma importancia para la institución, en tanto referente académico comprometido con el desarrollo local, regional y nacional en los campos de su especialidad, al ofertar y atender las demandas sociales y servicios con incidencia en los sectores de mayores necesidades. 


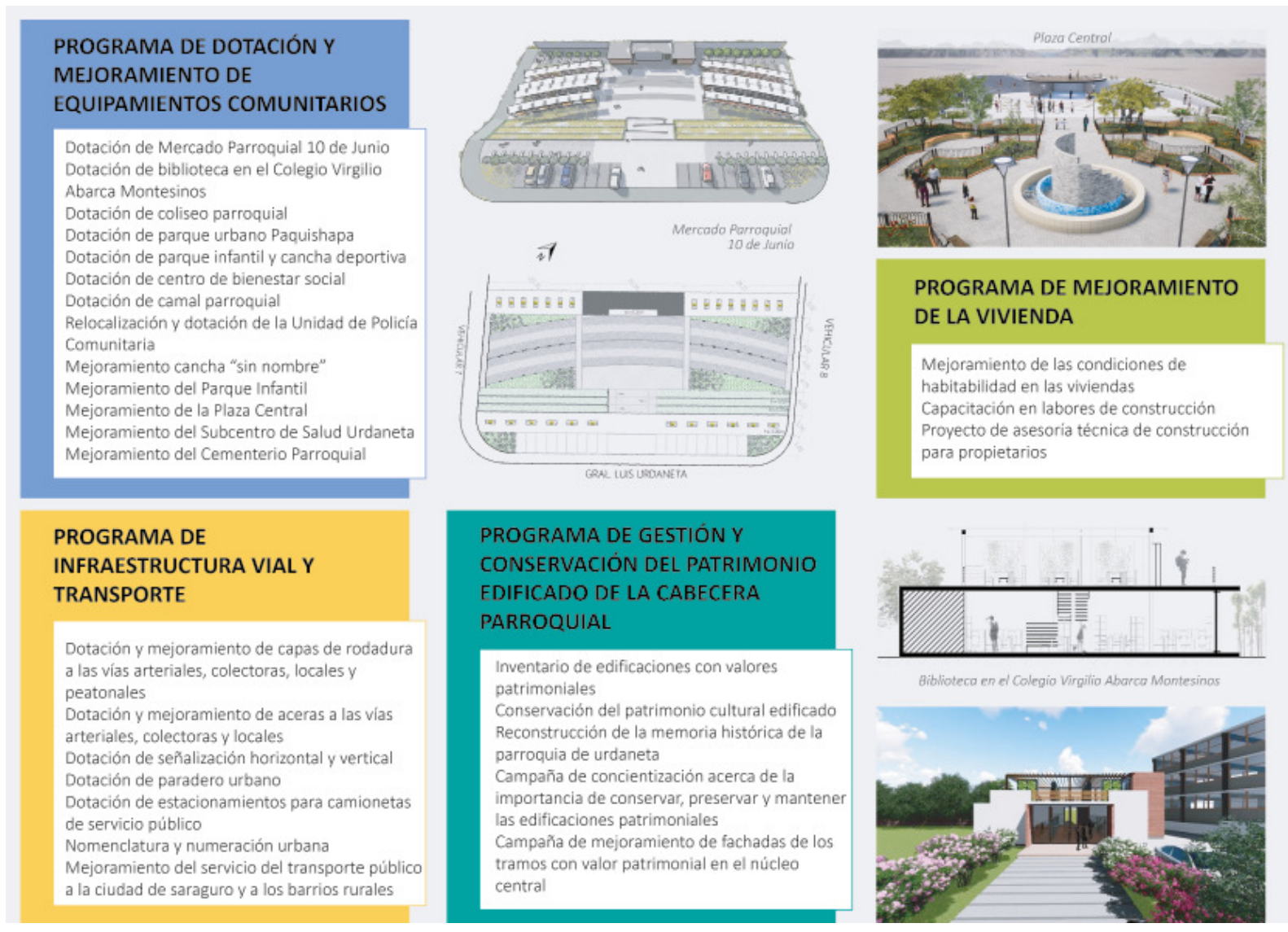

Figura 6. Programas, subprogramas y proyectos. Fuente: Elaboración de los autores en base a los datos y gráficos del curso de la Opción de Planificación Urbana septiembre 2018 - julio 2019.

\section{Referencias}

Baldwin, C. y Rosier, J. (2017). Growing Future Planners: A Framework for Integrating Experiential Learning into Tertiary Planning Programs. Journal of Planning Education and Research, 37(1), pp. 43-55.

Bermúdez, N., Cabrera, S., Carrión, A., Del Hierro, S., Echeverría, J., Godard, H. y Moscoso, R. (2016). La investigación urbana en Ecuador (19902015): cambios y continuidades. En P. Metzger, J. Rebotier, J. Robert, P. Urquieta y P. Vega Centeno (edits.), La cuestión urbana en la Región Andina. Miradas sobre la investigación y la formación, pp. 117-173. Quito: Pontificia Universidad Católica del Ecuador.

Capel, H. (2009). Las pequeñas ciudades en la urbanización generalizada y ante la crisis global. Investigaciones geográficas, (70), pp. 07-32.

Córdoba, M. (2014). Superar la fragmentación para ver el bosque. Documentos INTEC (21), pp. 181-204.

Costa, J. P., Ochoa, R. y Matos, M. (2015). Enfrentando las cuestiones urbanas. La enseñanza de la interdisciplinariedad por el proyecto de Urbanismo. On the waterfront, 34(5), pp. 5-24.

Frank, A. y Silver, C. (edits.). (2018). Urban Planning Education. Beginning, Global Movement and Future Prospects. Springer.

García, M., Villar, A., Fraile, P., Sánchez, N. y Márquez, J. (2018). Se hace Geografia al andar: la salida de campo itinerante y senderista. Didáctica Geográfica, (19), pp. 103-125.

Harden, R. (2000). The integration ladder: a tool for curriculum planning and evaluation. Medical Education, 34(7), pp. 551-557.

Henriquez, P. (ed.). (2018). Tendencias de la educación superior en América Latina 2018. UNESCO - IESALC y UNC.

Jaramillo, C. (ed.). (2014). Plan de Estudios Diseño Curricular de la Carrera de Arquitectura y Urbanismo de la Universidad de Cuenca - Ecuador. Cuenca, Ecuador: Universidad de Cuenca. 


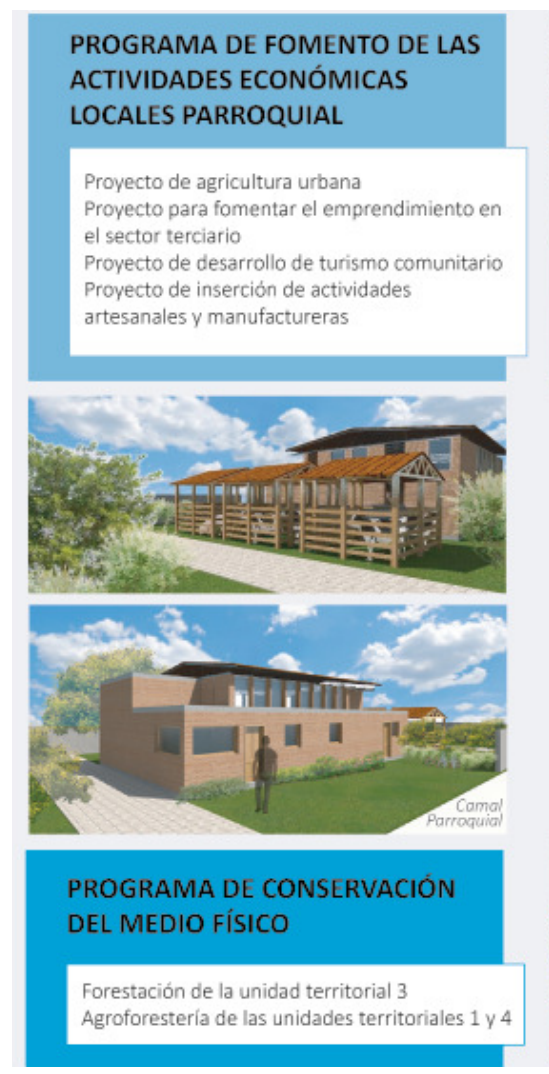

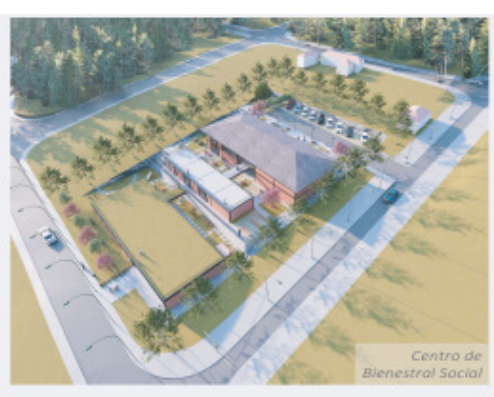

PROGRAMA DE INFRAESTRUCTURA Y SERVICIOS BÁSICOS

Implementación de canal de coronación Ampliación de cobertura del sistema de alcantarillado

Proyecto de mejoramiento del servicio de agua potable

Ampliación de cobertura del sistema de

recolección de desechos sólidos

Ampliación y mejoramiento del sistema de alumbrado público

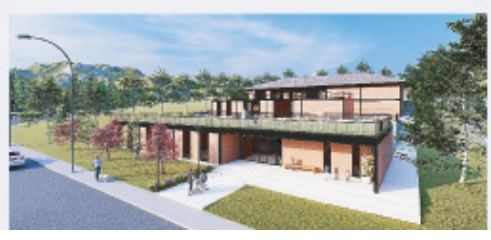

\section{PROGRAMA DE REESTRUCTURACIONES PARCELARIAS}

Proyecto de reestructuración parcelaria en las manzanas $01,02,03,04$ y 05 de la unidad urbanistica 05

Proyecto de reestructuración parcelaria en las manzanas 01,07 y 08 de la unidad urbanistica 06

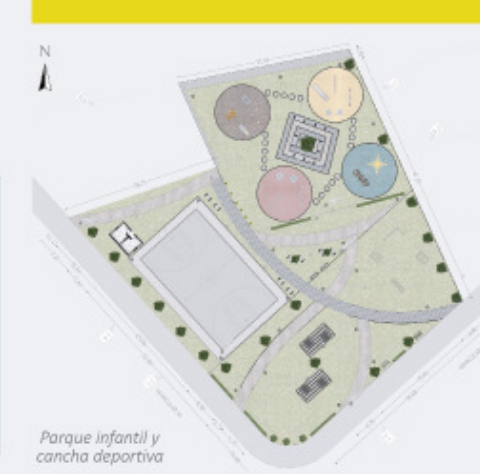

PROGRAMA DE FORTALECIMIENTO ORGANIZACIONAL PARA EL DESARROLLO LOCAL

Fortalecimiento organizacional

Proyecto de formación de líderes y liderezas

Kanai, M., Grant, R. y Jianu, R. (2018). Cities on and off the map: A bibliometric assessment of urban globalization research. Urban Studies, 55 (12), pp. 2569-2585.

Korobar, V. y Siljanoska, J. (2016). Challenges of teaching sustainable urbanism. Energy and Buildings, (115), pp. 121-130.

Menéndez, G. y Tarabella, L. (2017). El aprendizaje experiencial: una práctica de innovación que se afianza en la UNL. En I. Menéndez y Gustavo C.M., Integración, docencia y extensión 2: otra forma de enseñar y aprender, pp. 13-24. Santa Fe: Universidad Nacional de Litoral.

Molina, A. (2015). Extensión Universitaria, la función olvidada. CPU-e, Revista de Investigación Educativa (21), pp. 1-3.

Olmedo, N. y Farrerons, 0. (2017). Modelos Constructivistas de Aprendizaje en Programas de Formación. Barcelona: OmniaScience.

Pauta, F. (2013). Ordenación Territorial y Urbanística: un camino para su aplicación en el Ecuador. Cuenca: Universidad de Cuenca.

Reglamento de Régimen Académico. (21 de marzo de 2019). Registro Oficial. Quito.

Ruiz, J. (2002). La enseñanza del urbanismo y la enseñanza de la práctica del urbanismo. Un proyecto docente en el marco de la realidad urbana compleja. Madrid: Instituto Juan de Herrera.

Rumbach, A. (2016). Decentralization and small cities: Towards more effective urban disaster governance? Habitat International, (52), pp. 35-42.

Sánchez de Matrama, M. (2004). La extensión universitaria en Venezuela. Educere, 8(24), pp. 83-94.

Teal, M., Huang, C.-S. y Rodiek, J. (1998). Open space planning for Travis Country, Austin, Texas: a collaborative design. Landscape and Urban Planning, 42(2), pp. 259-268. 